\title{
COMPACT AND LONG VIRTUAL KNOTS
}

\author{
V. O. MANTUROV
}

\begin{abstract}
The theory of virtual knots is a generalization of the theory of classical knots proposed by Kauffman in 1996. In this paper solutions of certain problems of the theory of virtual knots are given. The first part of the paper is devoted to the representation of virtual knots as knots in 3-manifolds of the form $S_{g} \times I$ modulo stabilization. Using Haken's theory of normal surfaces, a theorem is proved showing that the problem of recognizing virtual links is algorithmically soluble. A theorem is proved stating that (any) connected sum of non-trivial virtual knots is non-trivial. This result is a corollary of inequalities proved in this paper for the (underlying) genus of virtual knots. The last part of this paper is concerned with long virtual knots. By contrast with the classical case, the classifications of long and compact virtual knots are different. Invariants of long virtual knots are constructed with the help of which one can establish the inequivalence of long knots having equivalent closures and also that some pairs of long virtual knots do not commute.
\end{abstract}

\section{INTRODUCTION}

Virtual knots were proposed by Kauffman in 1996 (see [35]) as a combinatorial generalization of classical knots regarded as equivalence classes of plane diagrams modulo generalized Reidemeister moves. A modification of the definition consists in the addition of a new type of crossing, called virtual, which is neither an underpass nor an overpass; it can be regarded as a random intersection of two branches of the knot which do not have to intersect but cannot diverge as a result of a local arrangement of the plane. For the generalized moves one considers Reidemeister virtual moves consisting of Reidemeister classical moves and also virtual moves that can be regarded as versions of a single "bypass" move; more precisely, the branch of the knot containing only interior crossings can be removed and resurrected anywhere else in the plane (with the same endpoints). A more detailed description of the theory of virtual knots can be found in [5, 15, 20, 21, 26, 36, 37, 38. Virtual knots can also be interpreted topologically as knots in "thickened surfaces" $S_{g} \times I$; the latter is the Cartesian product of a sphere with handles $S_{g}$ and the closed interval $I$, and the virtual knots are regarded to within isotopy and stabilization of these surfaces, that is, gluing and removal of new "thickened" handles which have empty intersection with the knot under consideration. In [19] Kuperberg showed that every virtual knot has a canonical minimal representation in $S_{g} \times I$ with minimal $g[1]$ A corollary of Kuperberg's theorem is that the classical theory of knots can be embedded in the theory of virtual knots in the sense that two classical virtual knot diagrams are virtually equivalent if and only if they give isotopic knots in the usual sense. The first proof of this fact was given in [7]. In the theory of virtual knots (which is

2000 Mathematics Subject Classification. Primary 57M25, 57M27; Secondary 57R65.

Key words and phrases. Classical knot, long virtual knot, classical crossing, virtual crossing, thickened surface, connected sum, long groupoid, Haken manifold, Reidemeister motion, destabilization.

${ }^{1}$ To be more exact, the direct product of a surface and an interval does not have to be connected in the case of a decomposing link $S_{g_{1}} \sqcup \cdots \sqcup S_{g_{k}}$ consisting of several components. But in this paper we consider only knots; therefore all surfaces will be deemed to be connected. 
considerably richer than the theory of classical knots) certain "non-classical" phenomena arise. For example, in the classical case the classification of ordinary (compact) knots coincides with the classification of long knots. This is not the case for virtual knots. Furthermore, long virtual knots do not commute; that is, the connected sum of two long knots $K_{1} \# K_{2}$ is not in general equivalent to the connected sum $K_{2} \# K_{1}$, and so on. In the papers $20,21,22,25,26,35,36,37$ and in many other papers, numerous invariants of virtual knots have been constructed.

The most widely known (see, for example, 20]) is the fact that the semigroup of (long) classical knots, defined with respect to a connected sum, has a good structure: classical knots commute, the decomposition of a knot into simple components is unique and finite, and there do not exist inverse elements for non-trivial classical knots.

The aim of this paper is to discuss the connection between long and compact (nonlong) virtual knots.

The construction of this paper is as follows. In the next section we give all the requisite definitions and statements of certain known results.

In the third section we prove that virtual knots are algorithmically recognizable.

In the fourth section we prove some inequalities relating to the genus of a virtual knot (in the sense of Kuperberg) and we prove that there is no non-trivial (long) virtual knot having an inverse, that is, such that the connected sum with it would give a trivial knot. We also study certain questions on long virtual knots.

The fifth section consists in the study of long virtual knots from the algebraic point of view. We construct certain variants of them enabling us, in particular, to show that some pairs of virtual knots do not commute (from which it follows that each of these virtual knots is non-classical). Kauffman's fundamental work [35] is devoted to a detailed discussion of the theory of virtual knots; that paper is a precursor to his book [15] and our work, as well as to the survey articles [16] and [5].

\section{BASIC DEFINITIONS AND KNOWN RESULTS}

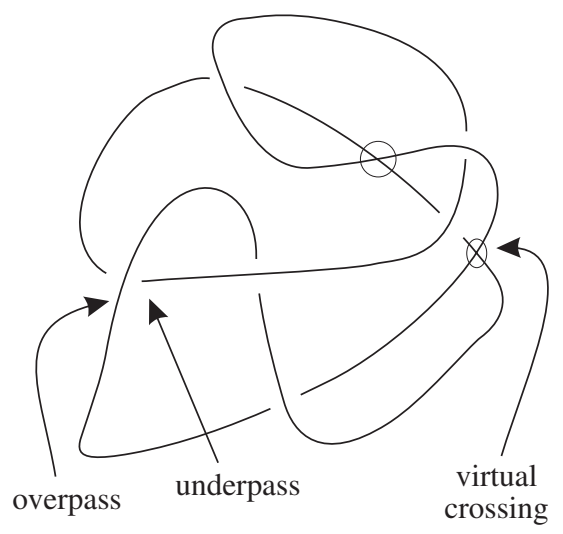

FiguRE 1. Virtual diagram

By the diagram of a virtual crossing (or, simply, virtual diagram) we mean a graph of valency four embedded in the plane $\mathbb{R}^{2}$, each vertex of which is either classical (in the sense that one branch (of the pair of opposite edges) forms an underpass and is depicted in the plane by a broken line, and the second branch is an overpass), or it is virtual (marked by a circle); see Figure 1. 
A virtual link is an equivalence class of virtual diagrams with respect to generalized Reidemeister moves (and also planar isotopies); see Figure 2.

Each of these motions means that the diagram changes locally as shown in Figure 2, elsewhere (in the part not depicted in the figure) the diagram can have an arbitrary appearance and is not altered under a Reidemeister transformation.

By a planar isotopy we mean a diffeomorphism of the plane onto itself preserving orientation.

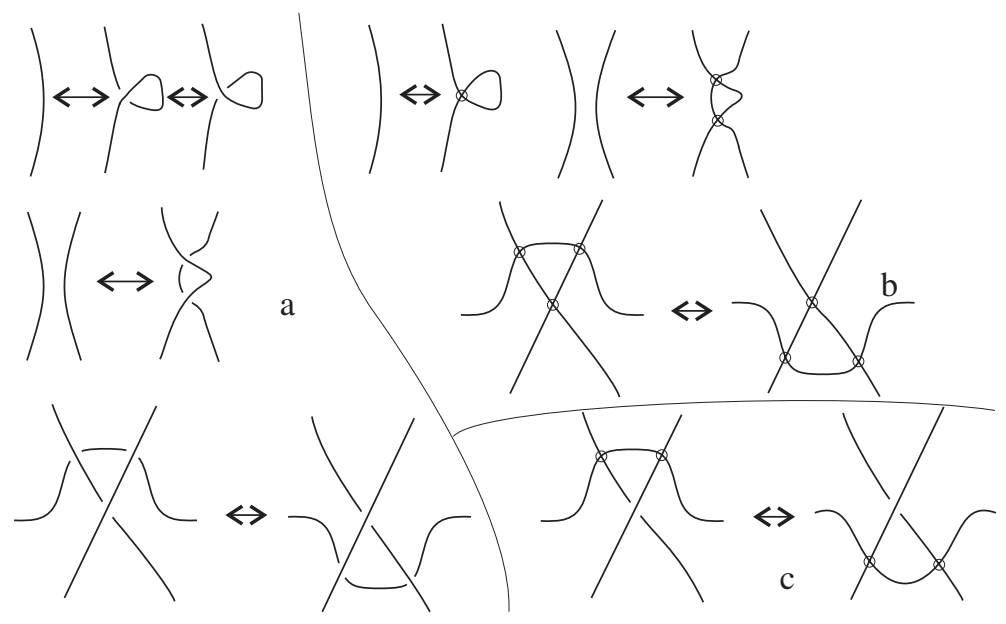

Figure 2. Generalized Reidemeister moves

The moves depicted in Figure 2 $\mathrm{a}$ are classical Reidemeister moves; the moves depicted in Figure 2b and Figure 2c can be (equivalently) changed by a bypass move, as indicated in Figure 3. A bypass move is described as follows: an arc containing successively several virtual crossings but not containing classical crossings can be transformed into any other arc with the same initial and end points; at the intersection points of the new arc with the rest of the diagram the knots become virtual crossings.

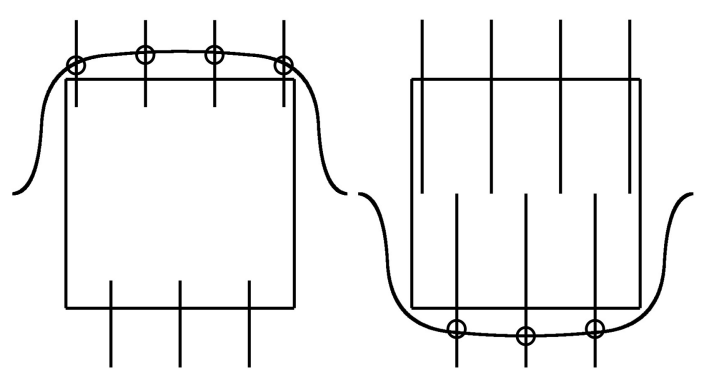

Figure 3. Bypass

A virtual diagram is said to be classical if it has no virtual crossings; a virtual link is said to be classical if it can be represented by a classical diagram. Historically the theory of classical knots appeared much earlier than the theory of virtual knots; it even goes back to the work of Gauss [6] and Vandermonde, while the combinatorial theory of classical knots was first made systematic by Reidemeister; two classical diagrams give the same classical link if and only if one of them can be converted to the other by 
classical Reidemeister moves. A natural question about equivalence relations on classical links arises: the classical and the new one defined by means of generalized Reidemeister moves. As it turns out, these relations are the same; that is, virtual Reidemeister moves "do not spoil" the ordinary equivalence of knots (see [7, 19]).

Remark 1. A trivial knot (link) is, as in the classical case, a knot (link) whose diagram has no crossings.

Let $K_{1}, K_{2}$ be two non-intersecting diagrams of oriented virtual knots on the plane $P$ with the property that some two-dimensional disc $E$ intersects $K_{1} \sqcup K_{2}$ in two $\operatorname{arcs}$ $A B \in K_{1}$ and $C D \in K_{2}$ with opposite orientations; that is, the first arc is oriented from $A$ to $B$, while the second is oriented from $C$ to $D$. Here when one goes round the circle $\partial E$ in a clockwise direction, the four points are encountered in the order $A, B, C, D$. We suppose further that there exists a line $\ell$ intersecting $E$ and not intersecting the diagrams $K_{1}, K_{2}$, so that the diagrams $K_{1}$ and $K_{2}$ lie on different sides of $\ell$. By the connected sum of the diagrams $K_{1}$ and $K_{2}$ (denoted by $K_{1} \# K_{2}$ ) we mean the diagram obtained from the diagram of the disjoint sum $K_{1} \sqcup K_{2}$ by removing the $\operatorname{arcs} A B$ and $C D$ and adding the arcs $D A$ and $C B$ with the orientations of the diagrams $K_{1}$ and $L_{2}$ extended to the diagram $K_{1} \# K_{2}$; see Figure 6. Note that the connected sum of virtual knots is not well defined. In Figures 4 and 5 we depict two inequivalent connected sums of a virtual trefoil with itself.

The equivalence class of a connected sum of two virtual knots is also not well defined. We will therefore use the term connected sum of virtual knots for any of the connected sums obtained as a connected sum of some of its diagrams.

The disjoint sum $L_{1} \sqcup L_{2}$ of two virtual links $L_{1}$ and $L_{2}$ is defined as the link having a diagram on the plane $P$ that does not intersect some line $\ell$ such that the subdiagram of it on one side of $\ell$ gives the link $L_{1}$, while the subdiagram with respect to the other side of $\ell$ gives the link $L_{2}$.

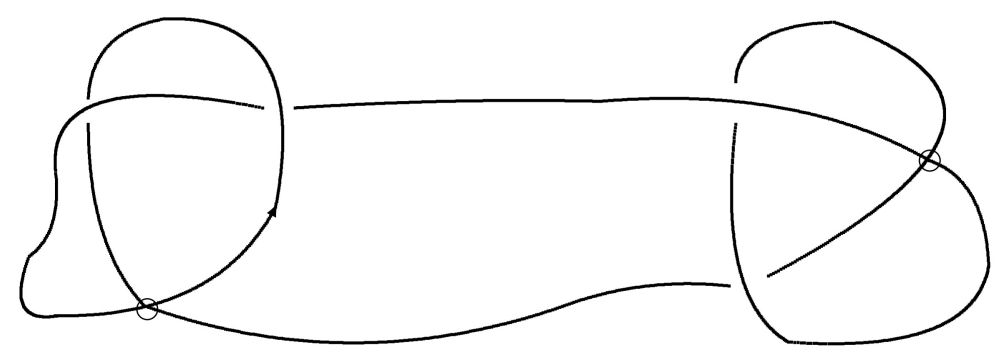

Figure 4. Connected sum $K \# K$

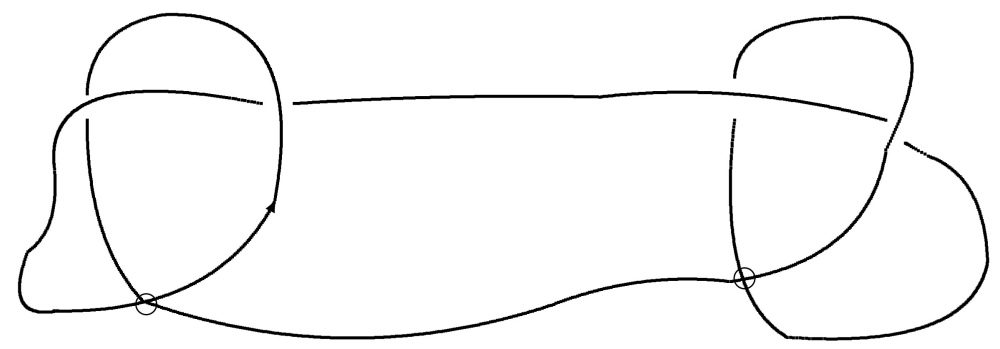

Figure 5. Another connected sum $K \# K$ 


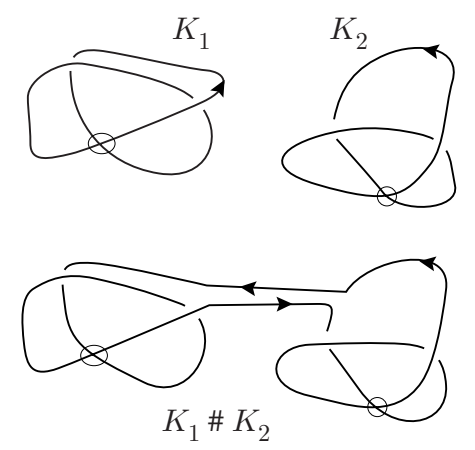

Figure 6. Example of a connected sum of virtual knots

We now turn to a topological definition of virtual knots (which is equivalent to the combinatorial definition given above). We consider an oriented closed two-dimensional surface $S_{g}$ with $g$ handles and the 3-manifold (thickened surface) $S_{g} \times I$, where $I$ is an interval 2 We shall study the theory of knots in $S_{g} \times I$. As in the case $\mathbb{R}^{3}=\mathbb{R}^{2} \times I$, the knots are coded by diagrams - generic projections onto the surface $S_{g}$, which are four-valent graphs. To recover the isotopy class of the knot at each crossing one has to indicate which branch is the upper one (forming the overpass) with respect to the coordinates given by the interval $I$. The local transformations establishing an isotopy between knots in the thickened surfaces are the same classical Reidemeister moves as in the case of classical knots in $\mathbb{R}^{3}$.

We now project $S_{g}$ onto $\mathbb{R}^{2}$ (so that the thickened surface $S_{g} \times I$ is projected onto $\mathbb{R}^{3}$ ). If we have a diagram (generic projection) of the knot into $S_{g} \times I$, then, by projecting it onto $\mathbb{R}^{2}$, again in general position, we obtain a four-valency graph. Some of its vertices are projections of crossings. We mark them as classical crossings with the same underpass-overpass information as before. The remaining vertices are intersection points of projections of edges (which were not intersected "above"). We mark them as virtual intersections.

Definition 1. The underlying genus of an indecomposable virtual link $L$ is the genus $g$ of the minimal surface $S_{g}$ such that the link $L$ is realizable in $S_{g} \times I$.

Remark 2. We note that in the classical theory of knots the genus of a knot, as put forward by Seifert, is widely used: this is the minimal genus of the covering surface. In what follows we shall indicate which genus we have in mind, the underlying genus or the Seifert genus, if it is not clear from the context.

The Reidemeister moves occurring on the surface generate precisely the generalized Reidemeister moves for the virtual knots on the surface.

Example 1. The virtual trefoil can be represented by a knot in the thickened torus $T^{2} \times I$; see Figure 7

Furthermore, some of the handles of our surface $S_{g}$ can be superfluous; that is, they have no common points with the diagram of the knot. In this case we can remove them. Conversely, we can add to each diagram of a knot on $S_{g}$ handles that do not affect the diagram so that the corresponding virtual knot is not altered. The addition of handles is called stabilization, and the removal of handles is called destabilization.

These discussions lead to the following well-known theorem; see, for example, 2 .

${ }^{2}$ For full generality one would also need to consider disconnected surfaces, but we shall not need this. 

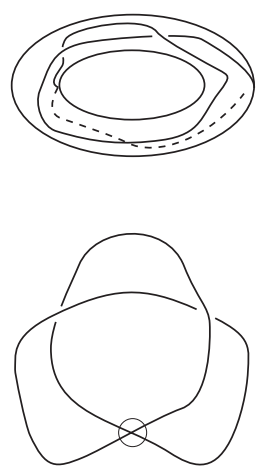

Figure 7. Diagram of a virtual trefoil on a torus

Theorem 1. Virtual knots (links) are knots (links) in abstract thickened surfaces considered to within isotopy and stabilization.

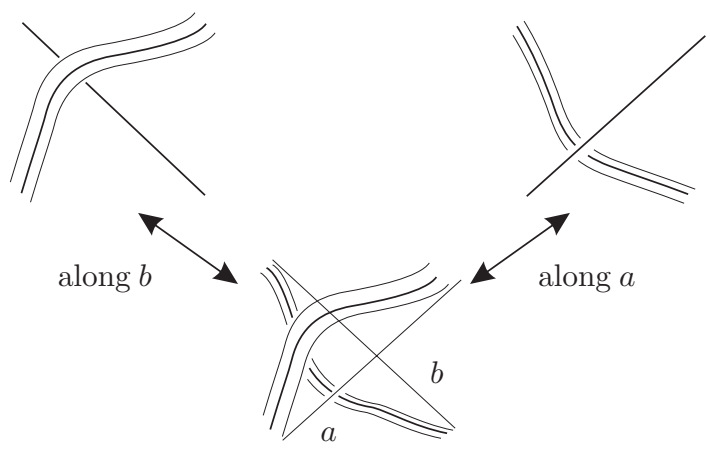

FiguRE 8. The placement of the handle is irrelevant

For a better understanding of this result we need to know how to "lift" virtual diagrams from the plane to $S_{g}$. This can be done as follows. If we have a diagram in the plane (or, which is equivalent, on the sphere $S^{2}=\mathbb{R}^{2} \sqcup \infty$ ), we can add handles in accordance with the location of the virtual crossings. Namely, for each virtual crossing we choose one of the two intersecting branches of the virtual knot, glue to its ends a handle and place this branch between the handles. Thus, on the manifold so obtained, the surface will have one virtual crossing fewer. Here there are four possibilities, depending on which of the two intersecting branches we convert to a handle and where we put this handle - above or below. The second option is inessential, since we construct a formal two-dimensional surface irrespective of what the embedding in three-space is. The addition of a handle along one branch $a$ in fact has the same effect as the addition of a handle along the other branch $b$ (intersecting $a$ in a virtual crossing): we can go from one picture to the other by a composition of stabilization and destabilization (that is, one can consider the addition of both handles and then remove one of them), as indicated in Figure 8 .

The application of the first Reidemeister virtual motion leads to the addition of a handle along which the knot passes with a twist. This means that before the destabilization of such a surface one must first apply a Dehn twist; this fact shows that the surface must be regarded as abstract and one must not fix a system of coordinates (say, give parallels) on it; see Figure 9. 

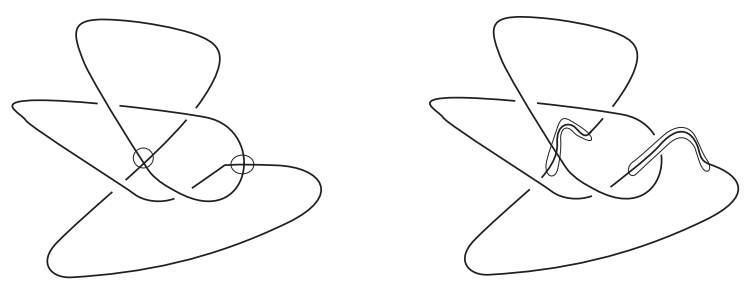

FIGURE 9. Virtual crossings corresponding to handles

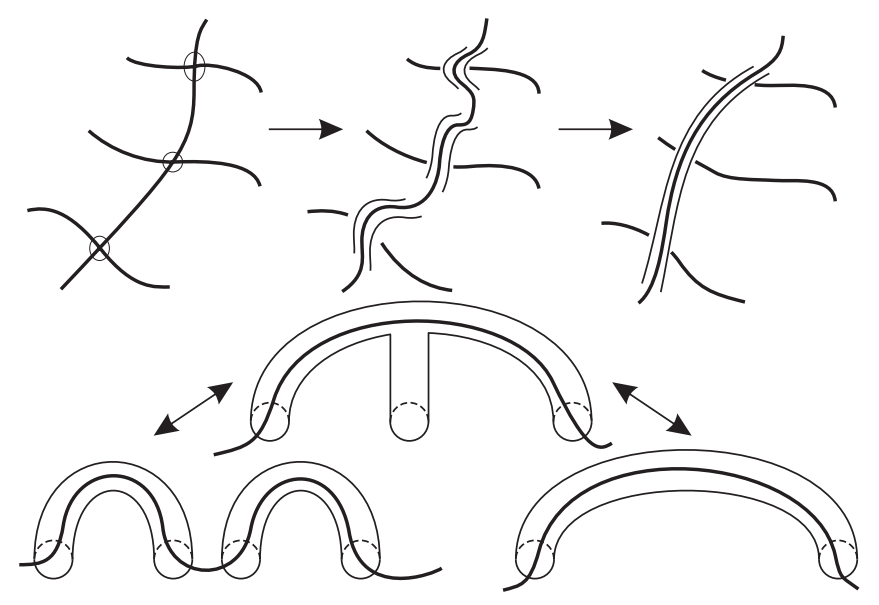

FIGURE 10. Bypass and stabilization

It is easy to see that the motion of a bypass is described as the surgery of several successive handles into one; see Figure 10. After this the handle must be transferred to another place and partitioned into handles in a new way.

The method of surgery of two successive handles into one via destabilization is depicted in the lower half of Figure 10.

Remark 3. It is also sometimes convenient to use disconnected surfaces and to allow the decomposition of $S_{g}$ into connected components $S_{k}$ and $S_{l}$ as possible destabilizations. But this happens only in the case when we work with decomposing links.

Theorem 2 (19). For each virtual link, represented as a link in a thickened surface, the minimal representation is unique. In other words, for each virtual link $K$ there exists a pair $\left(S_{\min }, K_{\min }\right)$ - a surface and a link - in $S_{\min } \times I$ (defined to within homeomorphisms of $S_{\min }$ onto itself taking the upper component of the boundary into the upper component of the boundary) such that any other pair $\left(S^{\prime}, K^{\prime}\right)$ giving the same link $K$ can be deformed into $\left(S_{\min }, K_{\min }\right)$ via destabilization and $\left(S_{\min }, K_{\min }\right)$ cannot be further destabilized.

In what follows we shall only be dealing with knots (with one component) so that surfaces will be assumed to be connected.

Kuperberg's theorem is also true for the case of tangles, that is, for objects analogous to virtual knots whose diagrams in the plane lie inside a strip $O y \times[0,1]$ and the intersection with the boundary of this strip consists of a finite (fixed) set of points: $m$ from the lower side $(O y \times\{0\})$ and $n$ from the upper side $(O y \times\{1\})$; here the intersections at these points are transversal and inside the strip the diagram looks the same as the 
V. O. MANTUROV

diagram of an ordinary virtual knot. We choose as equivalence, Reidemeister moves and homeomorphisms of the strip fixing points in a neighbourhood of its boundary. In this case we say that we have a (virtual) $(m-n)$-tangle.

We shall return to a detailed discussion of the important special case of virtual tangles, that is, (1-1)-tangles or long virtual knots.

\section{Virtual KNots ARE ALGORITHMiCALLY RECOGNIZABLE}

Classical links are algorithmically recognizable; this follows from a number of theorems. First, the addition to each classical link (that is not the disjoint sum of a trivial knot and some link) is a so-called Haken manifold (defined below). The main step in the recognition of classical links is the algorithmic recognizability of Haken manifolds. The latter result is a consequence of the work of many mathematicians, among whom one must single out Haken [8], Hemion [9] and Matveev [29]; in Matveev's book he gives an impeccable proof of all the main theorems of Haken's theory.

As an application, here we give the main statements of Haken's theory and, based on them, we prove the following result.

Theorem 3. There exists an algorithm that, given two virtual links $L_{1}$ and $L_{2}$ (defined, for example, by plane diagrams), determines whether or not they are equivalent.

This theorem was first presented as a preprint; see 27.

We shall need the well-known result that all three-dimensional manifolds can be triangulated. In what follows we shall assume that every three-dimensional manifold under consideration is endowed with some triangulation.

We shall be dealing with three-dimensional manifolds (with boundary) and twodimensional surfaces in them. All our manifolds are assumed to be oriented.

In each triangulated three-dimensional manifold $M$ (perhaps with empty boundary) we consider proper two-dimensional surfaces unless otherwise stated.

Definition 2. A surface $F$ is said to be proper if $F \cap \partial M=\partial F$. One can single out from the proper surfaces the so-called class of normal surfaces.

Normal surfaces are proper surfaces that, roughly speaking, behave normally with respect to the triangulation. In this sense the definition depends on the triangulation, although in what follows the properties of normal surfaces described by us are universal.

Among the normal surfaces we select the class of fundamental or basic surfaces from which all normal surfaces are obtained by applying geometric summation (see [29]).

The notions of normal and fundamental surfaces generalize to manifolds with a pattern - a graph without isolated points - on the boundary.

The main assertions of Haken's theory of normal surfaces consist in the following:

(1) The set of fundamental normal surfaces is finite and can be constructed algorithmically.

(2) For many natural properties $a$ the following assertions hold: if the manifold $M$ has a normal surface $F$ with property $a$, then there also exists a fundamental surface having property $a$.

It is clear from these facts that the search for a normal surface having "natural" properties is an algorithmically soluble problem.

Among the properties in item 2 of Haken's main assertions is, for example, the following: the property that a surface $F$ be a sphere giving a non-trivial element of the second homotopy group of the manifold $M$. Another such property is, for example, the possibility of destabilization for a given representative of a virtual link (see below). 
We shall not give a precise definition of a normal surface and a fundamental surface. Our proof of Theorem 3 will be based on Kuperberg's theorem and a number of theorems from the theory of normal surfaces, the proofs of which can be found in Matveev's book [29].

We shall need some further definitions.

A manifold $M$ is said to be irreducible if each embedded sphere in $M$ encloses a ball in $M$.

We shall use the definition of virtual knots as knots in thickened surfaces $\mathcal{M} \times I$ considered to within stabilization/destabilization. Here $\mathcal{M}$ is a compact (not necessarily connected) two-dimensional surface. Here we require that for each connected component $\mathcal{M}_{i}$ of $M$ the manifold $\mathcal{M}_{i} \times I$ should contain at least one component of the link $L$.

A realization of a virtual link is said to be minimal if it cannot be destabilized.

Thus, in order to compare virtual links one must know how to find their minimal representations and compare them. The algorithm which we give below uses 3-manifolds with a pattern on the boundary (see the definition below) which are connected with virtual links.

We shall use the following definitions and theorems from the Haken-Matveev theory; see 29$]$.

By a contracting disc for a surface $F$ in a 3 -manifold $M$ we mean an embedded (improper) disc $D \subset M$ intersecting $F$ on the boundary of the disc, that is, $D \cap F=\partial D$.

A (possibly disconnected) surface $F \subset M$ is called contractible in one of the following cases:

(1) It admits a contracting disc $D$ such that the curve $\partial D$ does not bound the disc on the surface $F$.

(2) There exists a ball $B$ such that $B \cap F=\partial B$.

A surface is incontractible if it is not contractible.

A surface $F \subset M$ is called boundary-contractible if there exists an (improper) disc $D^{2} \subset M$ such that $D^{2} \cap(\partial M \cup F)=\partial D^{2}$; here $D^{2} \cap F$ is a non-trivial arc in $F$ (that is, an arc not cutting off a disc from the surface $F$ ).

Next, a manifold $M$ is said to be boundary-irreducible if for each proper disc $D \subset M$ its boundary $\partial D$ bounds a disc in $\partial M$.

Suppose that we are given a 3-manifold. Then by a pattern (the concept was first introduced by Johansson; see [10]) we mean a fixed 1-polyhedron (graph) without isolated vertices on the boundary of the manifold (we suppose that this graph is a subpolyhedron of the fixed triangulation).

The existence of a pattern on the boundary does not change the definition of an incontractible surface nor that of an irreducible manifold.

The concepts of boundary-incontractible surface and boundary-irreducible manifold generalize to the case of manifolds with a pattern on the boundary in the following way.

A disc $D \subset M$ is said to be pure if it does not intersect a pattern on the boundary.

For boundary-irreducibility we require that each pure proper disc cuts off a ball (not intersecting a pattern). A boundary-incontractible surface $F$ is a surface such that for each pure (improper) disc $D^{2}$ such that $D^{2} \cap(\partial M \cup F)=\partial D^{2}$ and $\ell=\partial D^{2} \cap F$ is an arc in $F$, the arc $\ell$ cuts out a pure disc from the surface $F$.

Let $F$ be a surface in the manifold $(M, \Gamma)$ with a pattern on the boundary.

An oriented 3-manifold $M$ is said to be sufficiently large if it contains a proper incontractible boundary-incontractible surface different from $S^{2}$ and $D^{2}$.

It is natural to consider the notion of a sufficiently large 3-manifold together with the properties of irreducibility and boundary-irreducibility. This leads us to the following definition. 
A connected 3-manifold without boundary (therefore without a pattern on the boundary) is called a Haken manifold if it is irreducible, boundary-irreducible and sufficiently large.

A connected irreducible boundary-irreducible 3 -manifold $(M, \Gamma)$ with pattern $\Gamma$ on the boundary is called a Haken manifold if it is either sufficiently large or if the pattern $\Gamma$ is non-empty (so that $\partial M$ is also non-empty) and $M$ is a handlebody but not a ball.

Definition 3. Let $M$ be an irreducible boundary-irreducible 3-manifold. A proper annulus $A \subset M$ is said to be inessential if it is parallel (relative to $\partial$ ) to some annulus on the boundary $\partial M$ or if the axis of this annulus $A$ is contractible in the manifold $M$; otherwise the annulus $A$ is said to be essential.

An essential annulus $S_{g} \times I$ (relative to a manifold from which a tubular neighbourhood of the link has been removed, see later), two components of which lie in different components of the boundary $S_{g} \times\{0\}$ and $S_{g} \times\{1\}$ is precisely the type of annulus along which we can carry out destabilization.

In the case of several components, a manifold is called a Haken manifold if each of its components is a Haken manifold.

We shall require the following result.

Proposition 1 (Jaco-Rubinstein-Thompson; see, for example, [29, 34]). Every connected irreducible 3-manifold with non-empty boundary is either sufficiently large or a handlebody.

In what follows we shall be dealing with manifolds with a non-empty pattern on the boundary. In order for such a manifold to be a Haken manifold it suffices for us to verify that it is (more precisely, each connected component is) irreducible and boundaryirreducible, but not a ball.

Lemma 1 (Jaco-Rubinstein-Thompson; see, for example, [29, 34]). There is an algorithm recognizing whether a given manifold $M$ is reducible; if it is reducible, then the algorithm yields a 2-sphere $S \subset M$ not bounding a ball in $M$.

Proposition 2 (29]). Classical links are algorithmically recognizable.

This lemma follows from Haken's theory of normal surfaces; the proof is based on the following ideas; for each non-trivial indecomposable link the complement of an open tubular neighbourhood of it in $S^{3}$ is a Haken manifold. By endowing this manifold with a pattern on the boundary, we can recover the original link. After this the problem reduces to the problem of recognizing a Haken manifold; see [29] for details.

Proposition 3 (29]). There is an algorithm determining whether a given Haken manifold $M$ with pattern $\Gamma \subset \partial M$ on the boundary is a pure essential annulus. If there is such an annulus, then it can be constructed algorithmically.

We shall use this lemma to answer the question whether one can destabilize some realization of a virtual link.

Theorem 4 (29]). Given two Haken manifolds $(M, \Gamma)$ and $\left(M^{\prime}, \Gamma^{\prime}\right)$ with patterns on the boundary, there is an algorithm which can determine whether there is a homeomorphism of the first manifold onto the second taking the pattern $\Gamma$ to the pattern $\Gamma^{\prime}$.

We consider a virtual link $L$ and an arbitrary representation of it, that is, a pair $(M, L)$, where $M=\widetilde{M} \times I$ for some closed two-dimensional surface $\widetilde{M}$ and $L$ is a link in $M$ (for economy of notation we use the same letter $L$ to denote the original link and its realization in $M$ ). Let $N$ be a small open tubular neighbourhood of $L$. We cut out 
$N$ from $M$. As a result we obtain a manifold with boundary, which we denote by $M_{L}$. The boundary of this manifold consists of components of the boundary of $M$ (two if $M$ is connected) and several tori; the number of tori is equal to the number of components of the link $L$. We endow each torus with a pattern $\Gamma_{L}$ on the boundary: this pattern is a meridian of the corresponding component with three points on it: we add points so as to convert the circle into a graph - a subgraph of some triangulation. In this way we obtain a manifold $\left(M_{L}, \Gamma_{L}\right)$ with a pattern on the boundary.

Clearly the virtual link $L$ (and the pair $(M, L))$ can be recovered from $\left(M_{L}, \Gamma_{L}\right)$ since we know the method of constructing $M$ by gluing solid tori to the components of the boundary of $M_{L}$ where the meridians of these solid tori are distinguished by the pattern.

Lemma 2. Suppose that the link $L$ is not representable as a disjoint sum of a (nonempty) classical link and a virtual link. Then the manifold $\left(M_{L}, \Gamma_{L}\right)$ with pattern $\Gamma_{L}$ is a Haken manifold.

Proof. We can assume without loss of generality that the manifold $M_{L}$ is connected. In fact, we consider the connected components of $M$ and if the link $L$ does not have split classical components, then each of the components of the manifold $\left(M_{L}, \Gamma_{L}\right)$ is a Haken manifold; hence, so too is $\left(M_{L}, \Gamma_{L}\right)$ by definition.

We consider the connected case. In view of Proposition 1 it suffices for us to show that this manifold (or any of its connected components) with a pattern on the boundary is irreducible and boundary-irreducible; by definition, it cannot be a handlebody.

In the case $g=0$ we are dealing with classical links. Suppose that $g>0$. Then for each connected orientable two-dimensional surface $S_{g}$ the manifold $S_{g} \times I$ is irreducible. Thus, if the link $L$ is not classical (that is, $g \neq 0$ ), then if $N(L)$ is an open neighbourhood of it, the manifold $\left(S_{g} \times I\right) \backslash N(L)$ will be reducible if and only if it contains a sphere bounding a ball in $S_{g} \times\{0,1\}$ containing some non-empty set of components of the link $L$. This means that these components form a classical sublink of $L$ detached from the remaining components. This contradiction proves reducibility.

Next, since $L$ is not a disjoint sum of a trivial knot with some virtual knot, the manifold $M_{L}$ is boundary-irreducible.

Indeed, each curve in $S_{g} \times\{0\}$ or in $S_{g} \times\{1\}$, which can bound a disc in $S_{g} \times I$, is contractible to the boundary. Thus, boundary-reducibility can occur only when there is a proper disc with boundary lying in one of the tori, i.e., the boundary of one of the cut-out solid tori. This means that the component of the link corresponding to this solid torus is a split trivial knot.

Thus, the corresponding manifold is irreducible and boundary-irreducible and is therefore (by Proposition 1) a Haken manifold.

We now turn to the proof of the main theorem. Let $L, L^{\prime}$ be virtual links. The recognition algorithm consists of a successive application of the following four steps:

Step 1. Consider some realization $(M, L),\left(M^{\prime}, L^{\prime}\right)$ of virtual links $L, L^{\prime}$. We construct the manifolds with patterns on the boundary corresponding to them. We denote these by $\left(M_{L}, \Gamma\right),\left(M_{L^{\prime}}^{\prime}, \Gamma^{\prime}\right)$.

Step 2. We determine whether one of the manifolds $M_{L}$ or $M_{L^{\prime}}^{\prime}$ is reducible. If one of them is, then by Lemma 1 we can find a sphere not bounding a ball and in this way distinguish some split classical components of one (or both) links under consideration.

We make a change of notation: we denote by the old symbols $M, M^{\prime}$ manifolds obtained from $M, M^{\prime}$ by cutting out balls containing components of the link (followed by gluing balls in the holes). We also denote by $L, L^{\prime}$ those sublinks of the links $L, L^{\prime}$ that are left after removing the split classical components. 
Step 3. We determine (in accordance with Proposition 3) whether it is possible to apply destabilization to one of the manifolds $(M, L)$ or $\left(M^{\prime}, L^{\prime}\right)$. If it is possible, then we apply this destabilization and return to Step 2.

We apply Steps 2 and 3 as long as this is possible. Clearly this process will stop after a finite time: at each step we either remove some set of components of the links or we reduce the underlying genus of one of the surfaces.

Classical links are algorithmically recognizable. Thus, we can compare the separately standing classical sublinks of the links $L$ and $L^{\prime}$. If these two links are not isotopic, then we stop: the virtual links under consideration are not equivalent. Otherwise, we continue.

After applying the first three steps we reduce the problem to the case when the links have no split classical components and their realizations are minimal. So far, all the links are Haken manifolds (with a pattern on the boundary) by Lemma 2

Step 4. Each connected component of the manifolds $\left(M_{L}, \Gamma\right)$ and $\left(M_{L^{\prime}}^{\prime}, \Gamma^{\prime}\right)$ is a Haken manifold with a pattern on the boundary. Thus we can algorithmically solve the question whether there exists a homeomorphism $f: M_{L} \rightarrow M_{L^{\prime}}^{\prime}$ taking $\Gamma$ to $\Gamma^{\prime}$ (by Theorem 4). If such a homeomorphism exists, then the virtual links $L, L^{\prime}$ are equivalent. Otherwise they are inequivalent.

Thus the recognition problem is solved by carrying out the above four steps. Theorem 3 is proved.

Remark 4. The proof given above can also be carried out for the case of oriented virtual links and equipped virtual links.

\section{The UnDERLYING GENUS OF VIRTUAL KNOTS}

The aim of this section is to prove the following theorems.

Theorem 5. Let $K, K^{\prime}$ be two virtual knots. Then $g\left(K_{1} \# K_{2}\right) \geqslant g\left(K_{1}\right)+g\left(K_{2}\right)-1$; and in this case, if $g\left(K_{1}\right)=0$ or $g\left(K_{2}\right)=0$, then we have $g\left(K_{1} \# K_{2}\right) \geqslant g\left(K_{1}\right)+g\left(K_{2}\right)$.

As a consequence we get the following result.

Theorem 6. If $K_{1}$ and $K_{2}$ are virtual knots such that some connected sum $K_{1} \# K_{2}$ of them is trivial, then both knots $K_{1}$ and $K_{2}$ are trivial.

Indeed, if at least one of the virtual knots $K_{1}, K_{2}$ has an underlying genus greater than zero, then their connected sum has genus strictly greater than zero (by Theorem 5 ). In the case when both knots have genus zero we also need to take note of the following result.

Lemma 3. If $K_{1}$ and $K_{2}$ are two classical knots and $K_{1} \# K_{2}$ is some connected sum of genus zero, then it is equivalent to a (well-defined) classical connected sum $K_{1} \# K_{2}$.

This lemma follows directly from the proof of Theorem [5. The rest of Theorem [6 follows from the well-known fact that a connected sum of non-trivial knots is non-trivial; see, for example, $[3]$.

4.1. Two types of connected sum. Suppose that we are given two virtual knots $K_{1}$ and $K_{2}$ represented by knots in the thickened surfaces $M_{1} \times I$ and $M_{2} \times I$. Then there are two natural possibilities for representing their connected sum by a knot in a thickened surface.

The first way is as follows. We consider the thickened surfaces $\left(M_{1} \times I\right) \supset K_{1}$ and $\left(M_{2} \times I\right) \supset K_{2}$ and we cut out two vertical solid cylinders $D_{i} \times I$, where $D_{i} \subset M_{i}$, such 
that each of the sets $D_{i} \cap K_{i}$ is homeomorphic to an interval. We then glue the manifolds (identifying $\partial D_{1} \times I$ and $\partial D_{2} \times I$ in accordance with the orientations of the manifolds and the direction of the interval $I)$ and obtain $(M \times I)=\left(\left(M_{1} \# M_{2}\right) \times I\right)$ with a knot inside (denoted by $K_{1} \# K_{2}$ ).

Clearly, in this case, $g(M)=g\left(M_{1}\right)+g\left(M_{2}\right)$.

The second method of gluing the manifolds only works in special cases. Namely, we suppose that $K_{1}$ and $K_{2}$ lie in the manifolds $M_{1} \times I, M_{2} \times I$ where both genera $g\left(M_{1}\right)$ and $g\left(M_{2}\right)$ are strictly greater than zero. We suppose further that there exist non-trivial (not null homotopic) simple curves $\gamma_{1} \in M_{1}$ and $\gamma_{2} \in M_{2}$ such that for the cylinders $\Gamma_{i}$ homotopic to $\left(\gamma_{i} \times I\right)$ the intersection $\Gamma_{i} \cap K_{i}$ consists precisely of a single point (after a suitable homotopy these cylinders can be regarded as vertical). Then we can cut the manifolds $\left(M_{i} \times I\right)$ along the cylinders $\gamma_{i} \times I$ and then glue the resulting new components of the boundary ( $2 i$ pieces). As a result we obtain a manifold $M \times I$ for which $g(M)=g\left(M_{1}\right)+g\left(M_{2}\right)-1$ with some connected sum $K_{1} \# K_{2}$ lying in $M \times I$.

It turns out that by these two methods, in a certain sense all the essential possibilities for forming a connected sum of thickened surfaces in which three-dimensional manifolds lie are exhausted. Theorem 6 now follows from the above arguments.

4.2. Plan of the proof of Theorem 6. We consider the virtual knots $K_{1}$ and $K_{2}$ and some connected sum of them $K_{1} \# K_{2}$. We realize this connected sum as a knot in a thickened surface by the first method. We denote the corresponding surfaces by $M_{1}$, $M_{2}, M_{1} \# M_{2}$, and we denote the knots lying in these thickened surfaces by the same letters and by the same virtual knots: $K_{1}, K_{2}, K_{1} \# K_{2}$. We shall subject $\left(M_{1} \# M_{2}\right) \times I$ to transformations (destabilizations) and look at what happens to the knots. It suffices to construct the transformation process in such a way that the following conditions will hold at each moment:

(1) The ambient manifold $M$ can be divided into two parts $M_{1}, M_{2}$ that are manifolds with boundary such that each $M_{i} \times I$ contains the knot $K_{i}$ (with broken ends).

(2) The intersection $M=M_{1} \cap M_{2}$ consists of one or two components so that $\left(M_{1} \times\right.$ $I) \cap\left(M_{2} \times I\right)$ consists of one or two vertical annuli, that is, annuli having two components of the boundary on $\left(M_{1} \# M_{2}\right) \times\{0\}$ and $\left(M_{1} \# M_{2}\right) \times\{1\}$.

(3) The knot $K_{1} \# K_{2}$ intersects $\left(M_{1} \cap M_{2}\right) \times I$ at precisely two points; in the case when the intersection $M_{1} \cap M_{2}$ is disconnected, these two points of intersection must lie in different connected components of the set $\left(M_{1} \cap M_{2}\right) \times I$.

(4) The process stops when $g\left(M_{1} \# M_{2}\right)$ is the minimal genus of the knot $K_{1} \# K_{2}$.

If we prove the existence of such a process, then we shall have proved Theorem 6. Indeed, at each moment the virtual knots $K_{1}$ and $K_{2}$ are represented by knots in thickened surfaces of genera $g_{1}$ and $g_{2}$. The knot $K_{1} \# K_{2}$ lies in a surface of genus $g_{1}+g_{2}$ if the connected sum is of the first type, or in a surface of genus $g_{1}+g_{2}-1$ if the connected sum is of the second type. Thus, when the process stops we have either $g\left(K_{1} \# K_{2}\right)=g_{1}+g_{2}$ or $g\left(K_{1} \# K_{2}\right)=g_{1}+g_{2}-1$, where the latter can occur only when we have a connected sum of the second type at the end (and thus both numbers $g_{1}$ and $g_{2}$ are non-zero). Bearing in mind that on the left-hand side we have an underlying genus - the genus of a minimal surface for the connected sum, while $g_{i}(i=1,2)$ is the genus of some (not necessarily minimal) surface for $K_{i}$, we obtain the assertion of the theorem.

Furthermore, if at the end we obtain a representation for a connected sum of genus zero, that is, on the surface $S^{2} \times I$, then this representation will be the only way of forming the classical connected sum of the classical knots $K_{1}$ and $K_{2}$. Here we have used Lemma 3 . 
4.3. Destabilization process. In this subsection we describe a process which will complete the proof of Theorem 6 .

Suppose that, using the $i$ th method $(i=1$ or $i=2)$, we have constructed the connected sum of the knots $K_{1}$ and $K_{2}$. The main assertion is the following.

Assertion 1. If there is the possibility of destabilizing $M_{1} \# M_{2}$ with the knot $K_{1} \# K_{2}$ inside, then one of the following conditions holds:

(1) There is the possibility of destabilizing $M_{i}$, without affecting $M_{3-i}$ and the type of the connected sum. In this case we decrease the genus of the summand $M_{i}$ by one as well as the genus of $M_{1} \# M_{2}$, leaving the genus of $M_{3-i}$ unchanged.

(2) If we have a connected sum of the first type, then we go over to a connected sum of the second type, decreasing the genus of $M_{1} \# M_{2}$ by one and not changing the genus of the surfaces of $M_{1}$ or $M_{2}$.

(3) If we have a connected sum of the second type, then we go over to a connected sum of the first type decreasing each of the numbers $g\left(M_{1}\right), g\left(M_{2}\right), g\left(M_{1} \# M_{2}\right)$ by one.

Together with what we have discussed above, this process leads to a proof of Theorem 6.

Proof of Assertion 11. First we consider the case of a connected sum of the first type. We have $M=M_{1} \# M_{2}$. To simplify the notation we shall use the same letters for manifolds with boundary $\left(M_{1}\right.$ and $M_{2}$ as parts of $\left.M_{1} \# M_{2}\right)$ and manifolds without boundary $\left(M_{1}\right.$ and $M_{2}$ ). Let $D=M_{1} \cap M_{2}$.

Suppose that we can carry out a destabilization for $\left(\left(M_{1} \# M_{2}\right) \times I, K_{1} \# K_{2}\right)$. Then there exists a vertical annulus $C$ in $M_{1} \# M_{2}$ not intersecting the knot $K_{1} \# K_{2}$. If this annulus does not intersect $D$, then destabilization along $C$ relates only to one of the $M_{i}$ and does not affect $M_{3-i}$. This is the first case of Assertion 1 .

Suppose that any annulus $C$ along which we can carry out a destabilization intersects $D$. We shall consider only those annuli for which the intersection of $C$ and $D$ is transversal. In this case the annulus $C$ consists of finitely many components. Let $n$ be the minimal possible number of components of $C \cap D$.

Since $C$ and $D$ are proper submanifolds with boundary (vertical cylinders), their intersection can consist of:

(1) trivial circles;

(2) trivial arcs;

(3) horizontal circles;

(4) vertical arcs.

Here we call a circle trivial if it is a trivial element of the fundamental group of the annulus $C$; otherwise we call it horizontal. We call an arc trivial if it joins points on the same boundary component of the annulus; otherwise we call it vertical.

If the intersection $C \cap D$ has a trivial circle, then we can consider the innermost circle $\gamma=\partial \Delta$ (with respect to $C$ ). The interior of this circle contains no points of $D$. Since the disc $\Delta$ along with a disc in $D$ bounds a three-dimensional ball (see Figure 11) we can slightly modify the annulus $C$ so that the total number of components of the intersection between $C$ and $D$ is decreased and at the same time $C$ remains an annulus with nontrivial middle line (along which one can destabilize). The same situation arises in the case when we have a trivial arc; see Figure 12.

Next we state two auxiliary lemmas. 


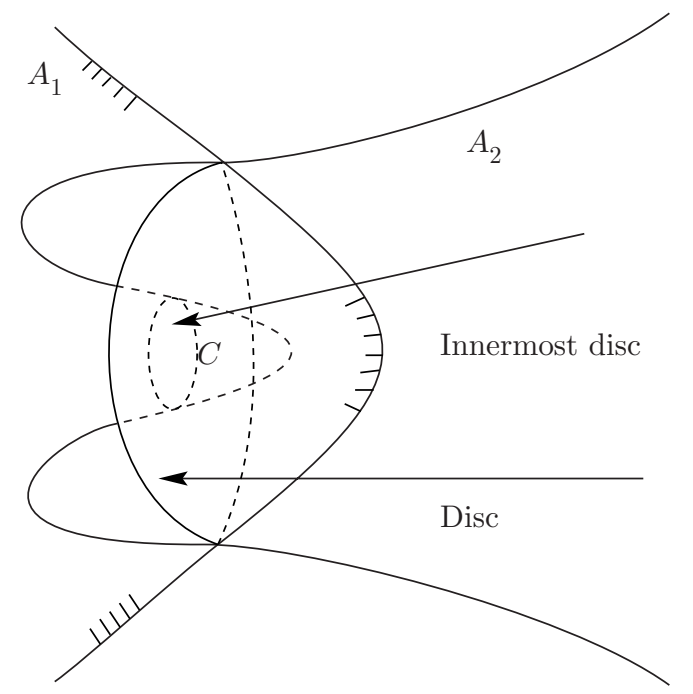

Figure 11. Non-horizontal arcs in $A_{1}$

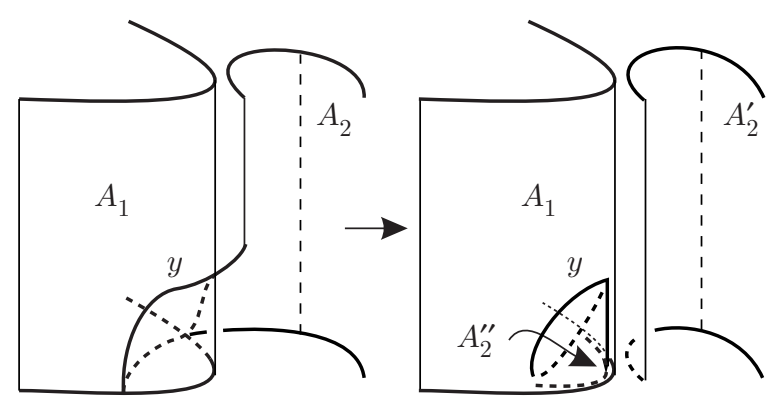

FIGURE 12. Moving crossings along non-vertical arcs

Lemma 4. Let $S_{g}$ be an orientable surface of genus $g$ and let $\Delta$ be a disc embedded in $S_{g}$. If a closed non-self-intersecting curve $\gamma \in S_{g} \backslash \Delta$ is trivial in $S_{g}$ and non-trivial in $S_{g} \backslash \Delta$, then it is parallel to $\partial \Delta$ (that is, $\gamma \cup \partial \Delta$ bounds a cylinder in $S_{g}$ ).

Indeed, if the curve $\gamma$, which has no common points with $\Delta$, bounds a disc in $S_{g}$, then $\gamma$ is contractible in $S_{g} \backslash \Delta$.

The next lemma is obvious.

Lemma 5. If a proper annulus $C^{\prime}$ is freely homotopic to an annulus $D$ in the class of proper annuli, then $C^{\prime}$ has a non-empty intersection with the knot $K_{1} \# K_{2}$.

Thus we can assume that the intersection $C \cap D$ either consists only of vertical arcs or only of horizontal circles (in general position the existence of a vertical arc contradicts the existence of a horizontal circle).

Suppose that the intersection consists only of horizontal circles. Then the annulus $C$ is homotopic to the annulus $D$ and, in accordance with Lemma 5 , the annulus $C$ intersects the knot $K_{1} \# K_{2}$. This leads to a contradiction.

Suppose now that $C \cap D$ consists only of vertical arcs. Then $C$ splits into $2 k$ parts $C_{1}, \ldots, C_{2 k}$, where the parts $C_{2 l+1}$ lie in $M_{1} \times I$, while the parts of the form $C_{2 l}$ lie in $M_{2} \times I$, where $l \in\{1, \ldots, k\}$. Thus the annulus $C \cap D$ splits into $2 k$ radial sectors (more 
precisely, radial segments); some of these sectors contain points of intersection with the knot; see Figure 13 (they are indicated by thick lines). We denote these radial segments by $r_{1}, \ldots, r_{2 k}$.

Next, each part $C_{i}$ of the annulus $C$ is incident to two radii $r_{j}$ and $r_{k}$. Here $D$ divides these radii into two parts, which we denote by $D_{j k}^{+}$and $D_{j k}^{-}$. Further, there are four possible versions of the answer to the following two questions:

(1) Is it true that each of the two parts $D_{j k}^{+}$and $D_{j k}^{-}$intersects the knot in exactly one point?

(2) Is it true that the annulus obtained by gluing $C_{i}$ to one of the parts $D_{j k}^{+}$or $D_{j k}^{-}$ cuts out a ball (so that if we glue $C_{i}$ to the other of these two fragments, then we obtain an annulus homotopic to $D)$ ?

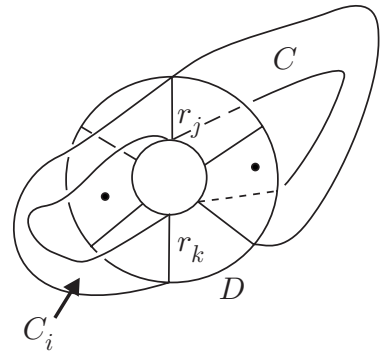

Figure 13. Annulus $C$ partitioned into sectors

Remark 5. Here we have in mind that a proper surface (for example, an annulus) $F \subset$ $M$ intersects a ball if $M \backslash F$ consists of two connected components, one of which is a topological ball. In other words, $F$ bounds a ball along with some part $P$ of the boundary $\partial M$ of $M$, so that $P \cup F$ is a sphere.

We consider the case when the answer to the first question is negative; that is, one of the two parts, say $D_{j k}^{+}$, does not intersect the knot (while the second part $D_{j k}^{-}$intersects the knot in two points).

If the annulus obtained by gluing $C_{i}$ to $D_{j k}^{+}$cuts out a ball, then we "extend" $D_{j k}^{+}$ across $C_{i}$; this decreases the number of components of the intersection of $C$ and $D$, which leads to a contradiction.

If the annulus obtained by gluing $C_{i}$ to $D_{j k}^{-}$cuts out a ball, then the annulus $C_{i} \cup D_{j k}^{+}$ is homotopic to $D$; thus it must intersect the knot. This again leads to a contradiction.

If both answers are affirmative, then again we come to a contradiction; a knot cannot intersect the boundary of a ball in exactly one point.

If both answers are negative, then one of the gluings $C_{i} \cup D_{j k}^{+}$or $C_{i} \cup D_{j k}^{-}$gives a non-trivial annulus not intersecting the knot. By a small perturbation we can make this annulus not intersect the bridge $D$. Thus we arrive at a contradiction, namely, that $C$ has the smallest intersection with $D$ among all annuli along which one can destabilize.

Finally, if, say, $D_{j k}^{+}$contains precisely one point of intersection with the knot $K_{1} \# K_{2}$, then the number of components of the intersection $C \cap D$ must be equal to two.

Indeed, if the number were greater than two, then it could be decreased by the method depicted in Figure 14. More precisely, from among the parts $C_{i}$ of the annulus $C$ we could choose just two and form from them a non-trivial annulus $C^{\prime}$ along which the thickened surface $\left(M_{1} \# M_{2}\right) \times I$ with knot $K_{1} \# K_{2}$ inside could be destabilized. 

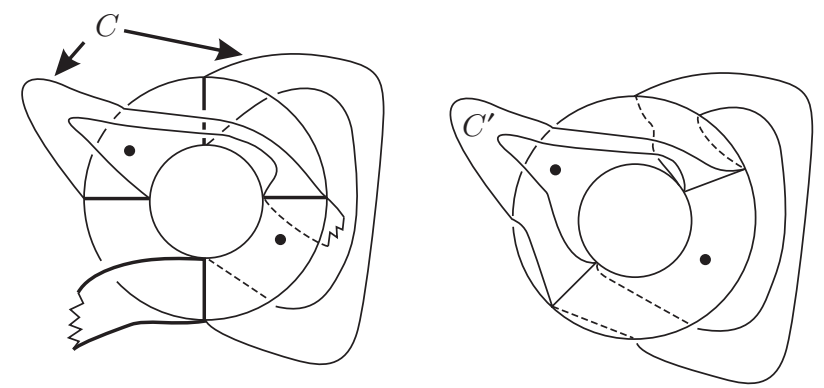

FIgURE 14. Simplification of the curve $C$

In other words, if we have more than two components in $C_{i}$, then we can find two of them and reglue them so that the annulus $C^{\prime}$ so obtained intersects the annulus $D$ in a smaller number of curves and therefore does not intersect the knot $K_{1} \# K_{2}$.

Thus, if the intersection $C \cap D$ is minimal (in the number of its components), then the number of components must be equal to two.

In this case, destabilization along such an annulus $C$ changes the type of the connected sum: after this we obtain a connected sum of the second type.

In fact, after such a stabilization, the disc $D$ splits into two parts and two of the points of intersection of the disc with the knot will be in different connected components.

The proof in the case when we have a connected sum of the second type (and destabilization converts it into a connected sum of the first type) proceeds in the same vein. Either there is the possibility of destabilizing one of the thickened surfaces $M_{1}$ or $M_{2}$ with the corresponding knot inside, or all the annuli along which one can carry out destabilization intersect $M_{1} \cap M_{2}$ (this set consists of two components). By considering an annulus having a minimal intersection with $M_{1} \cap M_{2}$ we arrive at a change of type of the connected sum (from second to first).

4.4. Discussion. We have proved that, as in the case of classical knots, no virtual knot, apart from the trivial knot, can have an inverse. However, certain questions remain unclear. First, it is not properly understood whether a knot can have an unbounded decomposition, that is, can there exist a virtual knot $K$ such that for each $n$ we have $K_{n 1} \# \cdots \# K_{n n}$ for some non-trivial virtual knots $K_{n 1}, \ldots, K_{n n}$ ? This does not contradict the estimates that we have obtained for the genus of a virtual knot, since all the $K_{n i}$ can have genus equal to one, as can the knot $K$ itself.

The answer to the above question is at present unknown; we conjecture that this cannot be the case. The answer to the following question is also unknown: can one have the equality $K=K \# L$ for some non-trivial virtual knots?

Furthermore, there are important questions about the commutability of virtual knots. For this it will be convenient to go over from ordinary (compact) virtual knots to long virtual knots.

\section{LONG VIRTUAL KNOTS AND THEIR INVARIANTS}

Long virtual knots give planar diagrams. The planar diagram of a long virtual knot is a smooth submersion in general position of the oriented line $\mathbb{R}$ into the plane $\mathbb{R}^{2}$ coinciding outside some sufficiently large disc with the identity embedding of the $O x$ axis; each crossing is either virtual or classical; in the latter case one indicates which branch goes above and which goes below (in the planar diagram the lower arc has a break). 
In what follows we will be looking at oriented long knots and will suppose that they are oriented from left to right along the $O x$ axis.

Two such submersions (diagrams) are said to be equivalent if one of them can be obtained from the other by a finite sequence of generalized Reidemeister moves, each of which is carried out inside a preassigned disc, and planar isotopies.

A trivial long knot is an equivalence class of diagrams having no crossings.

Long virtual knots admit the well-defined operation of taking the connected sum; for this one needs to place one of the long knots $K_{2}$ on an interval immediately to the right of the other long knot $K_{1}$. We shall use the same notation as for compact virtual knots: $K_{1} \# K_{2}$; by contrast with the case of classical knots this operation is unique for long knots.

Thus the semigroup $\mathfrak{W}$ of long virtual knots is well defined.

We present some new invariants of long virtual knots and demonstrate that with their help one can establish by simple means the non-triviality of various long virtual knots, as well as their non-commutativity (an effect that cannot happen in the classical case). These invariants are constructed starting from the combinatorics of the diagram of a long virtual knot and are algebraic objects generated by "arcs" - parts of the diagram - and certain formal operations. The invariance of such objects (with respect to Reidemeister moves) brings about the existence of certain formal operations.

Definition 4. By an arc of the diagram of a long virtual knot we mean a connected component of the set obtained by removing from it all virtual crossings, supposing that the branch forming the underpass is disconnected as is usually depicted in the plane.

In this sense an arc (if it is compact) starts and finishes either in a virtual crossing or an underpass, as long as it is forming overpasses. Furthermore, there are two unbounded arcs: a beginning one and an ending one, which can coincide in the case when there are no crossings.

The results of this section are published in abbreviated form in [26].

Next we give certain invariants of long virtual knots with the help of which we can answer questions of the following form:

(1) Given virtual knots $K_{1}$ and $K_{2}$ that have equivalent closures, are they equivalent?

(2) Do given long virtual knots $K_{1}$ and $K_{2}$ commute? In particular, if long virtual knots $K_{1}$ and $K_{2}$ do not commute, then neither of them can be classical.

In this section we describe a generalization of certain invariants of classical and virtual knots to the case of long knots. The main idea is the following. Suppose that we construct an invariant starting from the planar diagram of a knot by associating the knot with some algebraic object and associating parts of this diagram - elements of this object and crossings - with certain relations in this object (which we shall use to prove the invariance). Then in the case of long knots we have the following additional information:

(1) The initial and final (non-compact) arcs of the long knot play a special role of distinguished elements.

(2) There are two essentially different types of classical crossings: those in which the overpass is early (in accordance with the orientation of the long knot) with respect to the overpass and those in which the underpass is early.

These two observations are also applicable to the case of long classical knots, but they play a particularly striking role in the case of long virtual knots.

By having a diagram of a virtual knot (not a link) we can break it at some point and by stretching the ends to infinity obtain the diagram of a long virtual knot; see Figure 15. 


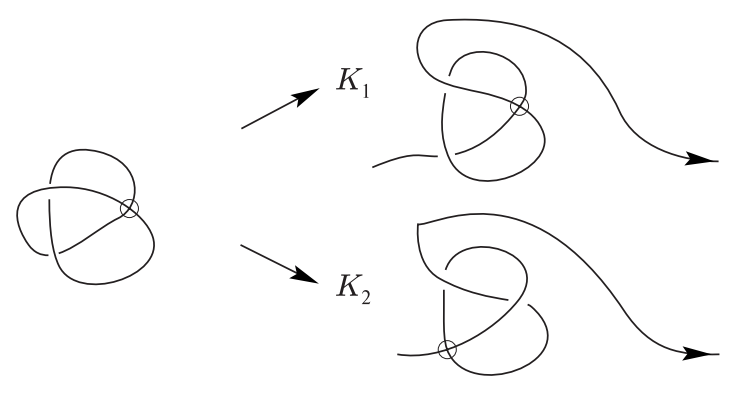

FiguRE 15. Breaking a virtual diagram

In the case of classical knots this operation is well defined; that is, it does not depend on the breaking point. In the case of long knots this is not so.

5.1. Long groupoid. We consider the following algebraic formalism (which is a generalization of the construction put forward in 21], which, in turn, is related to the constructions [11, 28]). Suppose that we are given a set $X$ with unary operations $f, f^{-1}$ and binary operations $\circ, /, *, / /$ satisfying the following axioms (the geometric meaning of which relates to Reidemeister moves):

(1) $\forall x \in X: x \circ x=x * x=x$.

(2) The operations $*$ and $\circ$ are distributive with respect to themselves and each other (on the right).

(3) $\forall x \in X$ we have $f\left(f^{-1}(x)\right)=f^{-1}(f(x))=x$.

(4) $\forall x \in X: f(x \alpha y)=f(x) \alpha f(y)$ for any operation $\alpha$ in the list $\{0, *, /, / /\}$.

(5) $\forall x, y, z \in X$ we have the relations:

$$
z \alpha(x \circ y)=z \alpha(x * y), \quad z \beta(x / y)=z \beta(x / / y),
$$

where $\alpha$ and $\beta$ are arbitrary operations in the list $\{0, *, /, / /\}$.

(6) $\forall x, y \in X:(x \circ y) / y=(x / y) \circ y=(x * y) / / y=(x / / y) * y=x$.

Relations of type 5 are called strange relations. It might be thought that each relation of type 5 (for example, $z \circ(x \circ y)=z \circ(x * y)$ ) leads to a contradiction (supposing that $\circ$ is not identically the same as the operation $*)$. However, as it turns out, this is not the case. The fact is that the operation $\circ($ as well as $*$ ) is invertible only on one side (on the left).

Any set endowed with the operations $f, \circ, *, /, / /$ with the properties described above is called a long groupoid (LG).

With each finite collection of generators we can associate a free long groupoid generated by this collection. To do this we need to take the generators $a_{1}, \ldots, a_{k}$, construct the set of admissible words, i.e., words obtained successively from the generators via the operations $\circ, /, *, / /, f$, and factor with respect to all the axiomatic relations of a long groupoid.

Consider the diagram $\bar{L}$ of a long virtual knot. We associate with its arcs elements $a=a_{1}, \ldots, a_{n}=b$ (here $a$ corresponds to the starting non-compact arc and $b$ the ending non-compact arc) and we choose them as the generators of a free long groupoid. We then factor this long groupoid by the following relations arising from the crossings of the diagram $\bar{L}$.

If two $\operatorname{arcs} u$ and $v$ are adjacent to a virtual crossing from opposite sides, where the arc $u$ is on the left with respect to an oriented arc coming out of this crossing and differing from $u$ and $v$, then we write the relation as $v=f(u)$. 
At each classical crossing there are three arcs. One of them (denoted by $y$ ) goes across the crossing, another (denoted by $x$ ) lies to the right of it, and a third (denoted by $z$ ) lies to the left. In this case, we write the relation $x \circ y=z$ for the corresponding elements if the arc $y$ occurs in the long knot earlier than the $\operatorname{arcs} x$ and $z$, or $x * y=z$ otherwise.

Having factored the free long groupoid generated by the elements $a_{1}, \ldots, a_{n}$ by the relations described above we obtain a long groupoid denoted by $M$; in the set $M$ we denote the starting and ending arcs of the long virtual knot (more precisely, their equivalence classes) by $a$ and $b$.

The main result of this subsection is the following.

Theorem 7. The triple $(M, a, b)$ constructed above and considered to within the natural isomorphism, is an invariant of long virtual knots under the hypothesis that the axioms described above hold.

It is obvious that for a trivial long knot $U$ the long groupoid is generated by the single element $a=b$.

Proof of Theorem [7. In the paper [21, a proof is given of the invariance of a virtual groupoid with the operation $\circ$. The proof of this invariance follows from the verification of all the Reidemeister moves. Now, in the case of a long groupoid with the operations $\circ$ and $*$ one must be aware of the type of the classical crossing encountered in the given Reidemeister transformation. In purely virtual Reidemeister moves, classical crossings are entirely absent. In the case of a semivirtual and first virtual move we have exactly one classical crossing and it has a definite type: either $\circ$ or $*$. Therefore the proof of the invariance of the triple $(M, a, b)$ is exactly the same as in the case with one operation $\circ$; one merely needs to bear in mind that $M$ is a virtual groupoid both with respect to the operation $\circ$ and with respect to the operation $*$. The same holds in the case of the second classical Reidemeister move: we have two classical crossings; moreover they both have the same type: either both use the operation $\circ$ or both use the operation $*$.

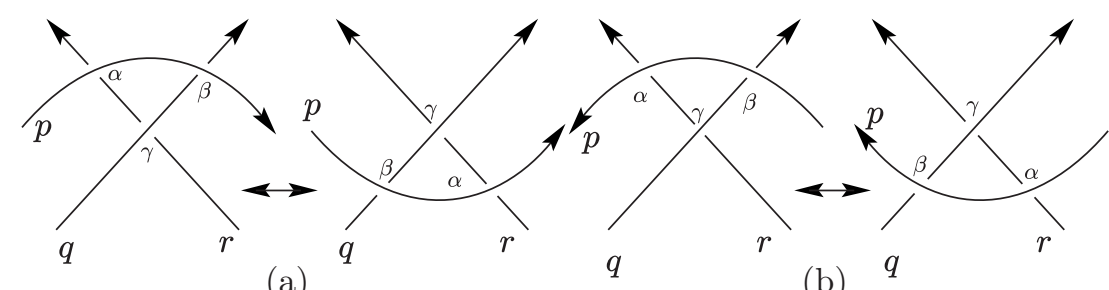

(a)

(b)

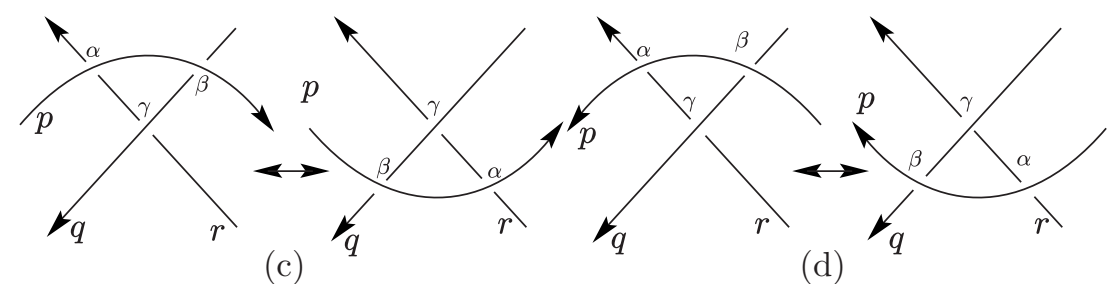

FIGURE 16. Verification of invariance with respect to the move $\Omega_{3}$

Thus the most interesting case is the third classical Reidemeister move in which at least one of the operations $\circ, /$ participates along with at least one of the operations $*, / /$. Obviously it suffices to consider only the cases indicated in Figure 16](a)-(d). 
In each of these cases we have three "entrances" and three "exits": the elements of the groupoid at the exit are expressed in terms of elements of the groupoid at the entrance. One needs to show that the expression does not change in the case when one applies the third Reidemeister move.

In each of the four cases (a)-(d) everything is in order with the generators $p$ and $q(p$ goes above, therefore at the exit we have a mark coinciding with the entrance mark, and the element $q$ is subjected to the action of $p$ equally in the case before the application of the Reidemeister move and in the case after the Reidemeister move). Thus the only case that remains to be verified is the exit corresponding to the entrance $r$.

In each figure, corresponding to each crossing there is an operation $\alpha, \beta$ or $\gamma$ from the list $\{0, *, /, / /\}$ (by applying this operation to the arc going below and to the underpass arc we obtain the corresponding overpass arc).

In the case (a) we have: each of the operations $\alpha, \beta, \gamma$ is "multiplication", that is, either $\circ$ or $*$.

Thus in the Reidemeister move depicted in the left upper corner we have: $(r \gamma q) \alpha p$ in the left half and $(r \alpha p) \gamma(q \beta p)$ in the right half. But, in view of the long-groupoid axiom we have $(r \gamma q) \alpha p=(r \alpha p) \gamma(q \alpha p)$. This last expression is equal to $(r \alpha p) \gamma(q \beta p)$ in accordance with the strange relation (since both $\beta$ and $\alpha$ are multiplication operations).

Next we consider the case (b). Here $\gamma$ is multiplication and $\alpha$ and $\beta$ are divisions. Thus we have the same equation:

$$
(r \gamma q) \alpha p=(r \alpha p) \gamma(q \alpha p)=(r \alpha p) \gamma(q \beta p) .
$$

The same equation holds also in the cases depicted in parts (c) and (d): one merely needs to note that both operations $\alpha$ and $\beta$ are either multiplications (as in the case (c)) or they are both divisions. The rest of the proof is obvious.

5.2. Examples. It is known that breaking the same compact virtual knot at different points leads in general to different (inequivalent) long virtual knots. A famous example is the Kishino knot (see Figure 17). The non-trivial knot depicted in the figure is obtained as the connected sum of two trivial knots. The non-triviality of the Kishino knot can be proved in various ways: by considering the Kauffman polynomial of the structure of the knot (see [18), using Fenn's approach using quaternionic bigroups ([1]) and considering the polynomial $\Xi$, which is a modification of the Jones-Kauffman polynomial put forward in 22 . In particular, it follows from the non-triviality of the Kishino knot that each of the two long knots forming it is non-trivial.

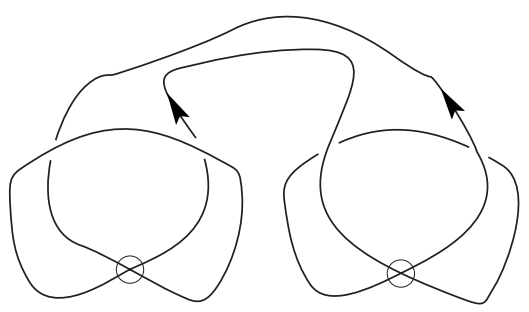

Figure 17. The Kishino knot

Consider the long virtual knots depicted in Figure 18. First we show that neither of them is equivalent to a trivial long knot. For this we use a linear long groupoid in the form of modules over the ring $\mathbb{Z}_{16}$ with the following structure:

$$
\begin{gathered}
x \circ y=5 x-4 y, \quad x * y=9 x-8 y, \\
f(x)=3 \cdot x .
\end{gathered}
$$




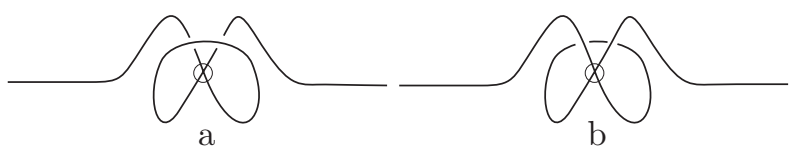

FiguRE 18. Two long virtual knots obtained by breaking a trivial knot

We show that for neither of them is the generator corresponding to the starting arc $a$ equal to the generator corresponding to the ending arc $b$.

For the first knot (Figure 18 a) we denote by $c$ the arc following after $a$. We have:

$$
9 a-8 \cdot(3 c)=c, \quad 5 b-4 \cdot(3 c)=c \quad \Longrightarrow \quad b=9 a .
$$
have:

For the second knot (see Figure 18b) we denote by $c$ the upper ("short") arc. We

$$
5 \cdot(3 b)-4 a=c, \quad 9 \cdot(3 a)-8 b=c \quad \Longrightarrow \quad b=9 a .
$$

For neither of these cases does the equality $a=b$ hold. Thus neither of the long knots depicted in Figure 18 is trivial.

If we consider the ring $\mathbb{Z}_{25}$ with the operation $a \circ b=6 a-5 b$ and $a * b=11 a-10 b, f(x)=$ $3 x$, we see that the two knots depicted in Figure 18 are in fact inequivalent: in the first case we have $a=11 b$, and in the second case $a=6 b$.

5.3. Long knots do not commute in general. It is known that any two classical knots commute. In this subsection we give an example of two long virtual knots that do not commute. This was first discovered in [26].

Theorem 8. Let $L, L^{\prime}$ be two long knots with $L$ classical (that is, we have a long diagram without virtual crossings). Then $L$ and $L^{\prime}$ commute.

Proof. In fact, we can make the long knot $L$ very small and pull it through $L^{\prime}$. In dragging it through the virtual crossings we have to apply a bypass move (or, which is equivalent, only pure virtual and semivirtual Reidemeister moves) (see Figure 19); when pulling through classical intersections we apply classical Reidemeister moves.

In the case of virtual knots we cannot carry out this proof because we cannot "pull" a line consisting of classical crossings through virtual crossings: we have obstructions to the motion.

We denote the knot depicted in Figure 18 by $K_{1}$ and the knot depicted in Figure 18b by $K_{2}$.

Theorem 9. The long virtual knots $K_{1} \# K_{2}$ and $K_{2} \# K_{1}$ are not equivalent.

Proof. We shall use the linear long groupoid represented by the module $M$ over the ring $\mathbb{Z}_{43681}=\mathbb{Z}_{11^{2} \cdot 19^{2}}$. We shall mark the elements of the long groupoid corresponding to the starting and ending arcs of the long knots under consideration.

We define the operations $\circ, *$ and the function $f$ as follows:

$$
\left\{\begin{array}{l}
a \circ b=t a+(1-t) b, \\
a * b=s a+(1-s) b, \\
f(a)=k a,
\end{array}\right.
$$

where $t=20, s=20+121 \cdot 19, k=70$.

The long-groupoid axioms are easily verified in this case; it suffices to note that $(t-1)(s-t)=(s-1)(s-t)$ in our module.

Consider the long knot $K_{1}$; we mark its diagram as shown in the upper half of Figure 20. 

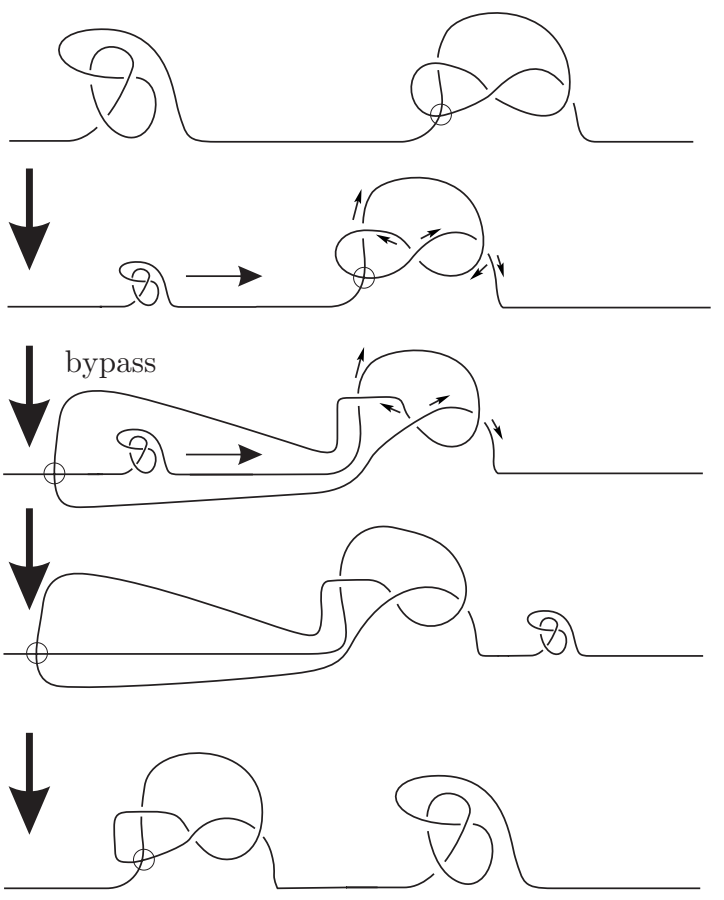

Figure 19. Classical (long) knots commute with all knots
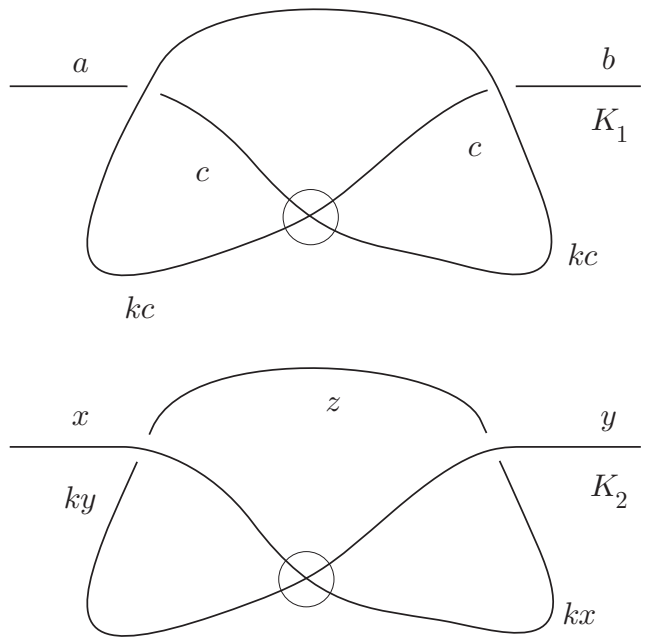

Figure 20. Marked knots $K_{1}$ and $K_{2}$

The equations giving the long groupoid of this knot are as follows:

$$
\left\{\begin{aligned}
s a+(1-s) k c & =c, \\
t b+(1-t) k c & =c
\end{aligned}\right.
$$


or equivalently,

$$
\left\{\begin{array}{l}
s a=(1+(s-1) k) c, \\
t b=(1+(t-1) k) c .
\end{array}\right.
$$

We have:

$$
\begin{aligned}
(1+(s-1) k) & =1+(19 \cdot 122) \cdot 70 \\
& =1+19 \cdot 70+19 \cdot 121 \cdot 70 \\
& =1331+19 \cdot 121 \cdot 70,
\end{aligned}
$$

which is divisible by 121 in the module of the knot $K_{1}$. Thus we see from the first equation of (3) that the initial element $a$ is divisible by 121 in view of the invertibility of the element $s$.

Since $a$ is a starting arc in $K_{1}$ the element of the module corresponding to the starting arc of any connected sum $K_{1} \# L$ is divisible by 121 . In particular, this holds for the long knot $K_{1} \# K_{2}$.

We claim that in general this is not the case for the knot $K_{2} \# K_{1}$.

Consider the knot $K_{2}$ in the lower half of Figure 20. The equations for the generators $x, y, z$ of the module are as follows:

$$
\left\{\begin{array}{l}
k t y+(1-t) x=z \\
k s x+(1-s) y=z
\end{array}\right.
$$

Thus, $z$ can be expressed in terms of $x$ and $y$, resulting in the single equation

$$
(k t+(s-1)) y=(k s+(t-1)) x .
$$

Hence

$$
k s+(t-1)=70 \cdot(20+121 \cdot 19)+19=162311 \equiv 88(\bmod 121) .
$$

The coefficient of $x$ in formula (5) is divisible by 11 but is not divisible by 121 .

We collect together the equations for the connected sum $K_{2} \# K_{1}$. The starting arc corresponds to the generator $x$. We must identify $y=a$ (since we are choosing a connected sum); in addition, we have the $\operatorname{arcs} b$ and $c$. Equations (51) and (3) together give

$$
\left\{\begin{aligned}
(k t+(s-1)) y & =(k s+(t-1)) x, \\
s y & =(1+(s-1) k) c, \\
t b & =(1+(t-1) k) c .
\end{aligned}\right.
$$

We denote the module so obtained by $\mathcal{M}$. We claim there is no element $T$ in it such that $121 \cdot T=x$.

Assume the contrary.

Consider the homomorphism $h: \mathcal{M} \rightarrow \mathbb{Z}_{(11 \cdot 19)^{2}}$ given by the maps

$$
\left\{\begin{array}{l}
x \mapsto 11 \cdot(19)^{2}, \\
y \mapsto 0, \\
b \mapsto 0, \\
c \mapsto 0 .
\end{array}\right.
$$

In fact, this map gives a module homomorphism. The second and third of equations (6) hold trivially since both sides are equal to zero. In the first equation we have zero on the left-hand side and $(k s+(t-1))\left(11 \cdot(19)^{2}\right)$ on the right-hand side. But as we have shown, $(k s+(t-1))$ is divisible by 11 ; therefore the right-hand side also vanishes.

By hypothesis, $121 \cdot h(T)=h(x)=11 \cdot(19)^{2}$ in $\mathbb{Z}_{11^{2} \cdot 19^{2}}$, which is impossible.

This contradiction completes the proof of the theorem. 
It follows from the fact that two long virtual knots do not commute that each of them is non-trivial and non-classical and they are inequivalent.

Finally we state the following conjecture for long virtual knots.

Conjecture 1. If $K$ and $K^{\prime}$ are two long virtual knots such that $K \# K^{\prime}$ and $K^{\prime} \# K$ are equivalent, then there exists a long virtual knot $L$, long classical knots $Q, Q^{\prime}$ and non-negative integers $m, n$ such that

$$
K=L^{m} \cdot Q, \quad K^{\prime}=L^{n} Q^{\prime},
$$

where $L^{m}$ denotes the connected sum of $m$ copies of the knot $L$.

5.4. Concluding remarks. We note that the linear model (described above) of a long groupoid is not unique. There exist other models of a long groupoid as well as other generalizations that lead to invariants of long virtual and classical knots. We content ourselves with giving just one explicit formula and some general arguments.

Let $G$ be a finite group, $k$ a positive integer coprime with the order of the group such that for any two elements $a, b \in G$ we have $a b^{k} a^{-1} b^{-k} \in Z(G)$, where $Z(G)$ is the centre of $G$.

Then the formulae $a \circ b=b a b^{-1}, a * b=b^{k+1} a b^{-k-1}$ define a long groupoid if one takes as the operation $f$ any automorphism of $G$.

Furthermore, one can consider the analogous formalism when, in the classical crossing, the mark on the arc passing overhead is subjected to a change: in this case we can assume that the marks are associated with the parts of the diagram of the knot at which it is partitioned by any crossings. Then at each classical crossing in the linear case we can consider the transition matrix: one can reconstruct the two upper marks from the two lower marks.

We shall not write down the axioms for such objects (they are called bigroupoids and virtual bigroupoids: see, for example, [5, 16, 17]). We note that for bigroupoids one can also construct a generalized formalism leading to invariants of long virtual knots.

\section{REFERENCES}

[1] A. Bartholomew and R. Fenn, Quaternionic invariants of virtual knots and links, www.maths.sussex.ac.uk (2003).

[2] J.S. Carter, S. Kamada and M. Saito, Stable equivalence of knots on surfaces, J. Knot Theory Ramifications 11 (2002), 311-322. MR1905687 (2003f:57011)

[3] R. H. Crowell and R. H. Fox, Introduction to knot theory, Springer-Verlag, Berlin-New York, 1977. MR0445489 (56:3829)

[4] H. Dye and L.H. Kauffman, Minimal surface representation of virtual knots and links, Algebr. Geom. Topol. 5 (2005), 509-535. MR2153118 (2006f:57005)

[5] R. A. Fenn, L. H. Kauffman and V. O. Manturov, Virtual knot theory: Unsolved problems, Fund. Math. 188 (2005), 293-323. MR2191949 (2006k:57011)

[6] C.F. Gauss, Zur Mathematischen Theorie der electrodynamischen Wirkungen, Werke Köningl. Gesell. Wiss. Göttingen 5 (1877).

[7] M. Goussarov, M. Polyak and O. Viro, Finite-type invariants of classical and virtual knots, Topology 39 (2000), 1045-1068. MR.1763963 (2001i:57017)

[8] W. Haken, Theorie der Normalflächen, Acta Math. 105 (1961), 245-375. MR0141106 (25:4519a)

[9] G. Hemion, The classification of knots and 3-dimensional spaces, Oxford Univ. Press, Oxford, 1992. MR.1211184 (94g:57015)

[10] K. Johansson, Homotopy equivalences of 3-manifolds with boundaries, Lecture Notes in Mathematics, vol. 761, Springer-Verlag, Berlin, 1979. MR0551744 (82c:57005)

[11] D. Joyce, A classifying invariant of knots, the knot quandle, J. Pure Appl. Algebra 23 (1982), no. 1, 37-65. MR0638121 (83m:57007)

[12] V.F. R. Jones, A polynomial invariant for links via Neumann algebras, Bull. Amer. Math. Soc. 129 (1985), 103-111. MR0766964 (86e:57006)

[13] S. Kadokami, Detecting non-triviality of virtual links, J. Knot Theory Ramifications 12 (2003), no. 6, 781-803. MR2008880 (2004j:57005) 
[14] L. H. Kauffman, State models and the Jones polynomial, Topology 26 (1987), 395-407. MR0899057 ((88f:57006)

[15] R. Fenn, L. H. Kauffman and V. O. Manturov, Virtual knot theory-unsolved problems, Fund. Math. 188 (2005), 293-323. MR.2191949 (2006k:57011)

[16] L.H. Kauffman and V. O. Manturov, Virtual biquandles, Fund. Math. 188 (2005), 103-146. MR2191942(2006k:57015)

[17] L. H. Kauffman and D. Radford, Bi-oriented quantum algebras and a generalized Alexander polynomial for virtual links, Diagrammatic morphisms and applications, Contemp. Math. 318, 113-140, American Mathematical Society, Providence, RI, 2003. MR.1973514 (2004c:57013)

[18] T. Kishino and S. Satoh, A note on non-classical virtual knots, J. Knot Theory Ramifications 13 (2004), no. 7, 845-856. MR2101229 (2005g:57018)

[19] G. Kuperberg, What is a virtual link?, Algebr. Geom. Topol. 3 (2003), 587-591. MR1997331 (2004f:57012)

[20] V. O. Manturov, Knot theory, Chapman \& Hall, Boca Raton, FL, 2004. MR.2068425 (2005d:57008)

[21] V. O. Manturov, Atoms and minimal diagrams of virtual links, Dokl. Math. 391 (2003), no. 2, 166-168. MR2042190 (2004k:57009)

[22] V. O. Manturov, An invariant polynomial of two variables for virtual links, Russian Math. Surveys 57 (2002), no. 5, 997-998. MR1992085 (2004b:57022)

[23] V. O. Manturov, Kauffman-like polynomial and curves in 2-surfaces, J. Knot Theory Ramifications 12 (2003), no. 8, 1145-1153. MR2017986 (2005h:57018)

[24] V. O. Manturov, Vassiliev invariants for virtual links, curves on surfaces and the Jones-Kauffman polynomial, J. Knot Theory Ramifications 14 (2005), no. 2, 231-242. MR.2128512 (2005k:57028)

[25] V. O. Manturov, Long virtual knots and their invariants, J. Knot Theory Ramifications 13 (2004), no. 8, 1029-1039. MR2108647 (2005i:57008)

[26] V.O. Manturov, Long virtual knots, Dokl. Math. 401 (2005), no. 5, 595-598. MR.2160133 (2006f:57006)

[27] V. O. Manturov, Virtual links are algorithmically recognisable, (2004) arXiv:math. GT/0408255 v1.

[28] S. V. Matveev, Distributive groupoids in knot theory, Mat. Sb. 119 (161) (1982), no. 1, 78-88; English transl. in Math. USSR-Sb. 47 (1984), 73-83. MR.0672410 (84e:57008)

[29] S. V. Matveev, Algorithmic topology and classification of 3-manifolds, Springer-Verlag, Berlin, 2003. MR.1997069 (2004i:57026)

[30] E. E. Moise, Affine structures in 3-manifolds. V. The triangulation theorem and Hauptvermutung, Ann. of Math. (2) 56 (1952), 96-114. MR0048805 (14:72d)

[31] W. Menasco and M. Thistlethwaite, The classification of alternating links, Ann. of Math. (2) 138 (1993), no. 1, 113-171. MR.1230928 (95g:57015)

[32] K. Murasugi, Jones polynomial and classical conjectures in knot theory, Topology 26 (1987), 187194. MR0895570 (88m:57010)

[33] T. Ohtsuki, Quantum invariants. A study of knots, 3-manifolds, and their sets, World Scientific, Singapore, 2002. MR1881401 (2003f:57027)

[34] A. Thompson, Thin position and the recognition problem for $S^{3}$, Math. Res. Lett. 1 (1994), no. 5, 613-630. MR 1295555 (95k:57015)

[35] L. H. Kauffman, Virtual knot theory, European J. Combin. 20 (1999), no. 7, 663-690. MR1721925 (2000i:57011)

[36] A. Bartholomew and R. Fenn, Quaternionic invariants of virtual knots and links, www.maths.sussex.ac.uk (2003).

[37] L. H. Kauffman, Detecting virtual knots, Atti. Semin. Math. Fis. Univ. Modena, Suppl. 49 (2001), 241-282. MR.1881100(2002k:57010)

[38] L. H. Kauffman, Diagrammatic knot theory (in preparation).

[39] L. H. Kauffman, Minimal surface representation of virtual knots and links (in preparation).

Eniseiskaya Ulitsa, 16/21-34, Moscow 129344, Russia

E-mail address: vassily@manturov.mccme.ru

Translated by G. G. GOULD 